\title{
Extreme action effects in reinforced concrete bridges from monitoring
}

\author{
Mark A. TREACY \\ $\mathrm{PhD}$ candidate \\ École Polytechnique \\ Fédérale de Lausanne, \\ EPFL, Switzerland \\ mark.treacy@epfl.ch
}

Mark Treacy, born 1983, received his degree in civil engineering from the National University of Ireland, Galway and his masters in structural engineering from the University of Surrey, UK. He previously worked as a structural engineer for the Arup group.

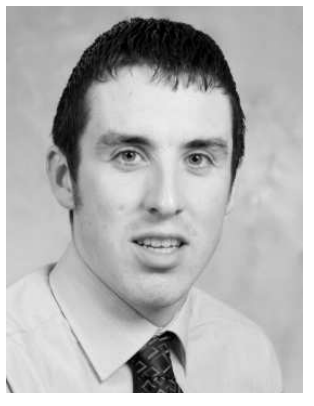

\author{
Eugen BRÜHWILER \\ Professor \\ École Polytechnique \\ Fédérale de Lausanne, \\ EPFL, Switzerland \\ eugen.breuhwiler@epfl.ch
}

Eugen Brühwiler, born in 1958, received his civil engineering and doctoral degrees from the Swiss Federal Institutes of Technology in Zurich and Lausanne. His research interests include examination of structural safety and UHPFRC for strengthening and rehabilitation of structures.

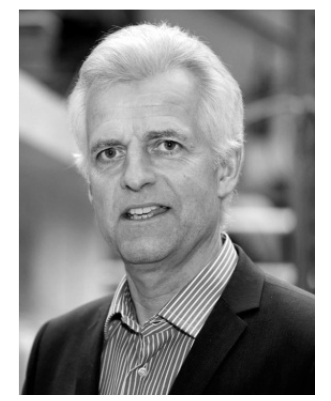

\section{Summary}

The need to maximise the lifetime of existing road bridges necessitates clear and efficient tools for engineers to perform structural safety verifications based on monitored data. This study develops methodologies for ultimate limit safety (ULS) verification of reinforced concrete elements using directly measured action effects. The paper incorporates results from a prestressed concrete highway bridge in Switzerland equipped with a structural health monitoring (SHM) system. The design considerations for the monitoring regime are presented. Estimates of the extreme traffic action effects for a given return period are obtained from daily block maxima results using a generalised extreme value approach. A number of factors are found to have a significant effect on the observed results, most notably the duration of monitoring.

Keywords: Bridge loading, box-girder bridges, monitoring, structural safety verification

\section{Introduction}

Heavy road freight transport volumes have increased dramatically in Europe in recent decades. For example there was an average annual increase in road freight transport (tonnes-km) of $31.2 \%$ in the EU-27 zone between 1995 and 2009 [1] and the same trends are present in many other countries. Such figures are alarming at first sight. But what does this mean for today's existing road bridges regarding structural and fatigue safety? The real answer lies in the action effects arriving in the structural elements, rather than the heavy vehicles causing them. Safety verification techniques incorporating measured action effects (e.g. strain, displacement, crack opening, acceleration, etc) as opposed to updated load models applied in structural analyses can lead to less conservative decisions being taken, which could otherwise result in costly interventions. In addition to removing uncertainty regarding traffic loading, direct monitoring of the bridge reduces further uncertainties regarding dynamic and environmental effects, in particular temperature effects, as they are inherently included in the measurements.

This paper presents results from an on-going research study of a prestressed box-girder bridge in Switzerland to understand the long term performance of the deck slab under traffic and environmental effects with the aid of monitoring. The focus is on the upper flange or deck slab of the girder which experiences the localised effects of all heavy axles at an elemental level, in this case steel reinforcing bars (rebars). The paper firstly describes some of the considerations in developing the monitoring system. The work then examines the nature of extreme traffic loading events in a number of rebars within the deck slab which are instrumented with strain gauges. Codified verification approaches require characteristic load effect values for a certain return period for ULS verification so techniques for extrapolation of measured action effect data, in this case strain, for the estimation of extreme behaviour are tested and the influence of monitoring duration is studied. 


\section{Direct monitoring of bridge action effects}

\subsection{Long term monitoring}

In literature relating to bridge load effect calculation there is much work on the simulation of measured traffic and subsequent extreme value estimation. However, extreme value estimation made from continuous measurement of bridge elements themselves receives less attention. Siegert et al [2] recorded 120 second signals for over 100 days for a prestressed concrete bridge and worked with weekly maximums for extreme value estimation. Pircher et al. [3] presented studies of minimum 8 weeks for 3 prestressed concrete bridges where the focus was on fatigue damage. In contrast this study includes datasets of over 200 days continuous high-frequency measurement capturing all traffic crossing a bridge. Only a few years ago, such measurements would not have been possible due to the limitations with data storage.

\subsection{Case study monitoring project}

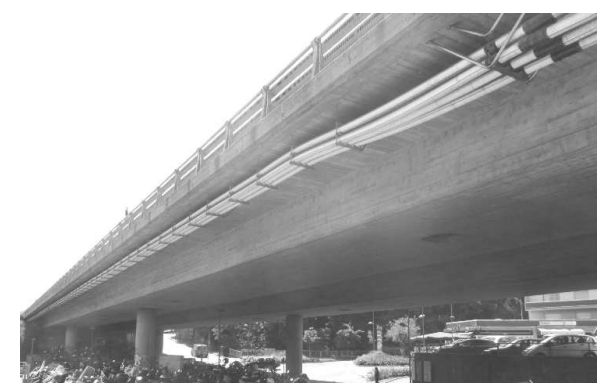

Fig. 1: Morges Highway Bridge
Constructed in 1963, the Morges Bridge (Fig. 1) is a 110.5 $\mathrm{m}$ long, three-span, twin box-girder structure located on the Swiss A1 highway between Geneva and Lausanne. Average daily traffic volumes are in the region of 70,000 vehicles. A monitoring system, described in detail in [4], was installed in 2011. The system comprises strain gauges on the uncracked concrete surface and steel rebars, thermocouples and accelerometers. This paper will focus solely on the direct measurement of 'action effects' i.e. strain in the deck slab rebars using multiple electrical resistance strain gauge measurements on four separate bars.

As the two girders in each direction are completely identical but separated structures only one girder was instrumented for this study. Two $10 \mathrm{~mm}$ diameter bars in the transversal direction and two $12 \mathrm{~mm}$ diameter bars in the longitudinal direction in the bottom layer of the deck slab reinforcement were instrumented. The three strain gauge arrangement on the transverse bars (S1a to S2c) shown in Fig. 2(b) capture the movement in the positive transverse bending moment in the deck slab which is dependent on a vehicle's transverse position. The gauges were installed after localised removal of the cover concrete below the bars.

(a)

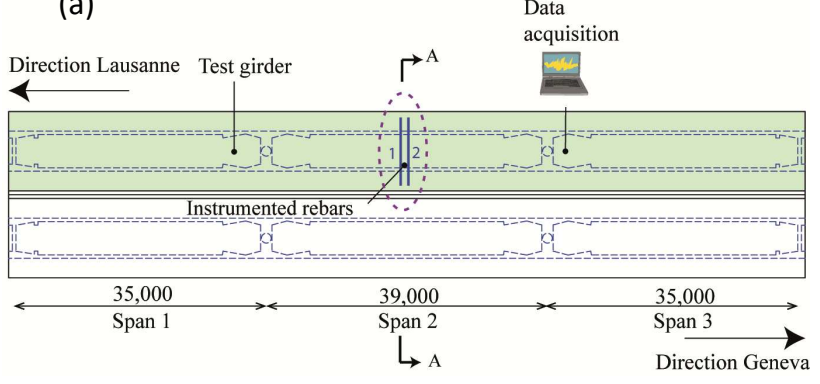

(b)

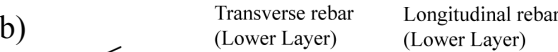

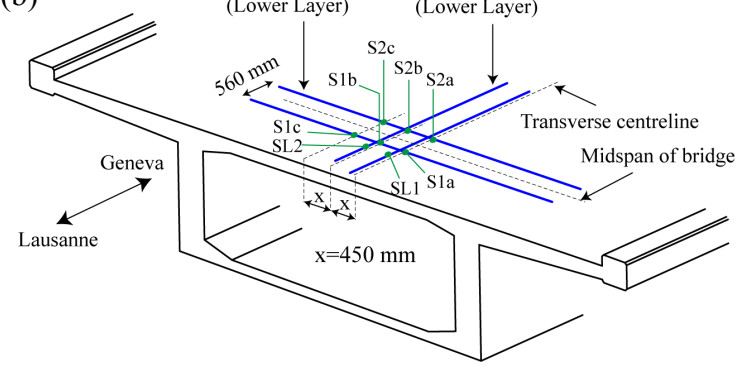

Fig. 2: (a) Bridge plan and; (b) Schematic 3D section showing instrumented rebars and gauge positions

A long-term monitoring campaign commenced in February 2012 and 200 days of data are analysed in this study. A sampling frequency of $50 \mathrm{~Hz}$ was used to record data continuously for 22 hours per day, stopping only between 22:00 and 01:00.

\subsection{Calibration and testing of system}

The monitoring system was calibrated using a series of 'soft load tests'. A special permission fiveaxle, 60 tonne, crane was used to perform test runs over the bridge at varying speeds and in different lane positions. A numerical simulation was performed using ANSYS software, to verify that the measurements system was behaving correctly and good agreement was found (Fig. 3(c)). 
(a)

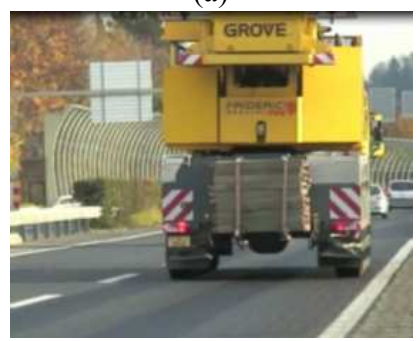

(b)

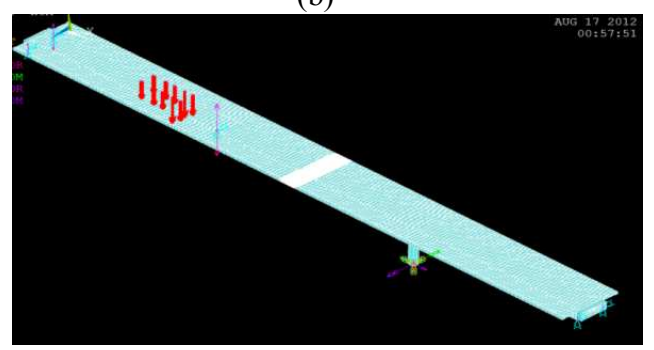

(c)

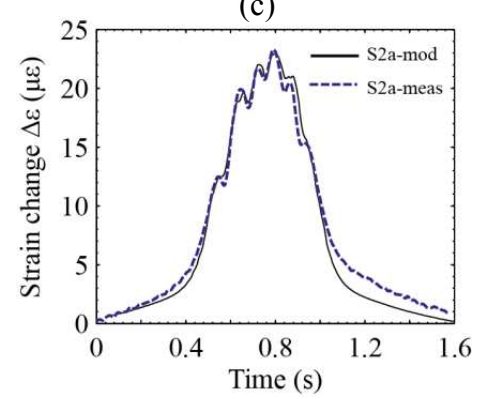

Fig. 3: (a) Soft load tests, (b) Simulation in ANSYS® and; (c) Comparisons of simulations and field test measurements for a transverse rebar under the passage of a test vehicle

\subsection{Choice of monitoring frequency}

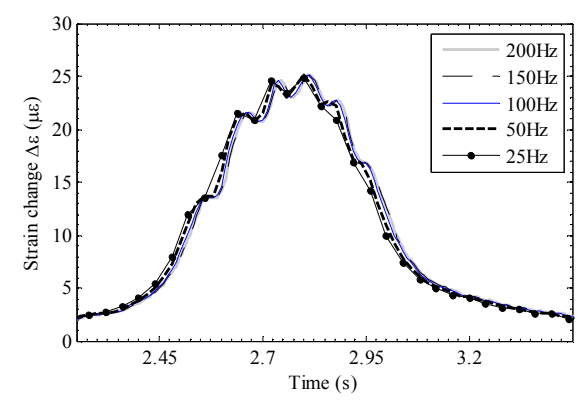

Fig. 4: Comparison of the same loading event with various sampling frequencies

A sufficient sampling rate must be chosen in order to avoid aliasing or under-sampling. When a continuous signal is sampled as a discrete time-series with a constant sampling time increment $\Delta \mathrm{t}$, the signal contents with any frequencies higher than the Nyquist frequency $\left(\mathrm{f}_{\mathrm{N}}=1 / 2 \Delta \mathrm{t}\right)$ cannot be accurately represented. i.e. the sampling rate must be twice the Nyquist frequency to perfectly reconstruct the original signal [5]. The initial choice (considering vehicle frequency) can be tested by reducing the sampling rate of a high frequency measurement and checking the signal deterioration as shown in Fig. 4 for a transverse rebar for a truck travelling at $85 \mathrm{~km} / \mathrm{h}$. It can be seen that a $25 \mathrm{~Hz}$ sampling rate is sufficient to capture the correct strain amplitude. In addition a low pass Bessel filter was implemented to attenuate signals with frequencies higher than a cut-off frequency.

\subsection{Decomposition of thermal and traffic effects}

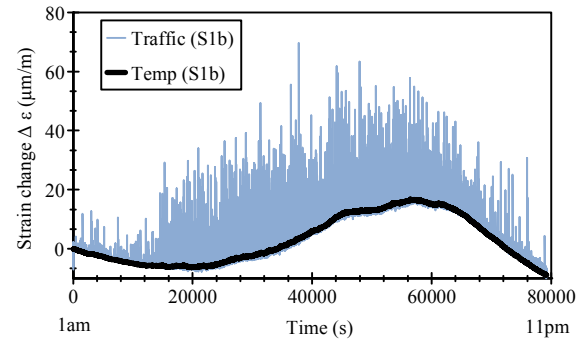

Fig. 5: Single day's strain measurement in transverse deck slab rebar
Temperature itself is an important load case with daily thermal 'waves' due to the heating up and cooling down of a structure present in strain signals. With high frequency measurement, this effect can be separated with a smoothing operation by replacing the original signal with the unweighted arithmetic mean value of that measurement and a specified number of neighbouring values, $\mathrm{n}$ as defined by the following equation [6] where $\mathrm{N}$ is the total number of data measurements:

$$
f\left(y_{i}\right)=\frac{1}{2 n+1} \sum_{k=i-n}^{k=i+n} y_{k} \quad \text { for } N-n>i>n
$$

The use of the function is shown in Fig. 5 on a daily strain signal from sensor S1b on a deck slab rebar recorded in April 2012. The traffic effect remains if the fitted thermal wave is subtracted from the original signal. In this way thermal effects and traffic effects can be analysed separately if there are no non-linear relationships present.

\subsection{Monitoring duration}

An important decision in planning of a monitoring system is the duration of monitoring period required for an accurate estimation of fatigue damage or extreme events. The preferable option is always more data but often a practical limit must be chosen. Maljaars et al. [7] suggested a period 
of approximately 3 weeks outside of public holidays to be representative of road traffic distribution for a whole year. Leander \& Karoumi [8] proposed a damage based approach of testing for convergence of monitored data for fatigue examination but literature is scarce regarding measurements of extreme bridge effects. This will be studied further in the following sections.

\section{Safety verification approaches}

\subsection{Action effect for Ultimate Limit State safety verification}

\subsubsection{Code of practice based approaches}

Eurocode 1-2 load model 1 for the design of new bridges in Europe specifies a design characteristic traffic action effect with a 1000 year return period or probability of exceedance of $5 \%$ in 50 years [9]. However, for examination of existing structures a more appropriate return period is required. In the case of existing bridges, return period values vary according to code formats. For example in the US, periods tend to be in the region of 2-10 years [10] while recent European approaches have used 75 years as in [11]. In the case of medium-term examination, a return period of 75 years has been proposed and will form the basis of this work. The notion of degree of compliance, $n$, is introduced for deterministic verification of the structural safety in the Swiss standards for existing structures [12] and is given by:

$n=\frac{R_{d, \text { updated }}}{E_{\text {d,updated }}}$

where $R_{d, \text { updated }}$ and $E_{d, \text { updated }}$ are the examination values of resistance and action effect, respectively. This formulation not only gives the information whether the structural safety is fulfilled, i.e. $\mathrm{n} \geqslant$ 1.0 , but also indicates by how much the verification is fulfilled (or not). The focus of this work is on the live load component of the updated characteristic action effect, $E_{k}\left\{Q_{\text {updated }}\right\}$. To obtain the examination value (assuming it is the main action), an appropriate partial factor for variable actions should be applied as follows:

$$
E_{d}\left\{Q_{\text {updated }}\right\}=\gamma_{Q} E_{k}\left\{Q_{\text {updated }}\right\}
$$

\subsubsection{Extrapolation of extreme values - procedure and theoretical background}

This study will use a block maximum approach fitted to a generalised extreme value (GEV) distribution to estimate assessment characteristic value $E_{k}\left\{Q_{\text {updated }}\right\}$ using the approach described in [13]. Given a number $n$ of independent random variables with a common distribution $F$, it can be proven that the largest $M_{n}=\max \left\{X_{1}, X_{2}, X_{3}, \ldots, X_{n}\right\}$ has the probability distribution function:

$$
\operatorname{Pr}\left\{M_{n} \leq z\right\}=\{F(z)\}^{n}
$$

In this case, $X_{i}$ could represent extreme action effects on a bridge .e.g. strain in an element so that $M_{n}$ represents the maximums of the process over $n$ time units of observation. This is not immediately useful as $\mathrm{F}$ is unknown but one possibility is to use statistical techniques to estimate $F$ from the observed data and to substitute this estimate into equation (4). Small discrepancies in the estimate of $F$ can lead to large discrepancies in $F^{n}$. An alternative approach is to take $F$ as unknown and look for families of models for $F$ based on the extreme data only. The Generalised Extreme value (GEV) distribution given in equation (5) combines the families of Gumbel, Fréchy and Weibull in which each one have different forms of tail behaviour of the distribution $F$. The Weibull function has a finite upper bound, while the other two are unbounded but show different rates of decay in the upper tail.

$$
G(z)=\exp \left\{-\left[1+\xi\left(\frac{z-\mu}{\sigma}\right)\right]^{-\frac{1}{\xi}}\right\}
$$

Where $\mu, \sigma$ and $\xi$ are the location, scale and shape parameters respectively and satisfy: $-\infty<\mu<\infty$, $\sigma>0$ and $-\infty<\xi<\infty$. For $\xi<0$ the GEV is equivalent to the type III (Weibull) EVD, $\xi>0$ represents the Type II (Fréchy) EVD, while the GEV becomes Type I (Gumbel) as $\xi$ approaches 0 . The GEV 
distribution parameters are determined using the maximum likelihood method as described in [13]. Under the assumption that $\left\{Z_{1}, Z_{2}, \ldots, Z_{n}\right\}$ are independent variables which follow the GEV distribution then the log-likelihood for the GEV parameters when $\xi \neq 0$ is:

$\ell(\mu, \sigma, \xi)=-m \cdot \log \sigma-\left(1+\frac{1}{\xi}\right) \sum_{i=1}^{m} \log \left[1+\xi\left(\frac{z_{i}-\mu}{\sigma}\right)\right]-\sum_{i=1}^{m}\left[1+\xi\left(\frac{z_{i}-\mu}{\sigma}\right)\right]^{-\frac{1}{\xi}}$

provided that:

$1+\xi\left(\frac{z_{i}-\mu}{\sigma}\right)>0$, for $i=1, \ldots, m$

The case of $\xi=0$ involves separate treatment using the Gumbel limit of the GEV distribution and leads to the following log-likelihood:

$\ell(\mu, \sigma)=-m \cdot \log \sigma-\sum_{i=1}^{m} \log \left[\left(\frac{z_{i}-\mu}{\sigma}\right)\right]-\sum_{i=1}^{m} \exp \left\{-\left(\frac{z_{i}-\mu}{\sigma}\right)\right\}$

Maximisation of equations (6) to (8) with respect to the GEV parameters $(\mu, \sigma, \xi)$ provides the maximum likelihood estimate with respect to the whole GEV family. Manipulation of the Gumbel distribution cdf gives the following expression known as the 'Standard Extreme Variate' (SEV) which expresses the data in Gumbel probability paper format:

$\frac{1}{\sigma\left(z_{i}-\mu\right)}=-\ln \left(-\ln \left(p_{i}\right)\right)$

Originally proposed by Gumbel, the following quantile formulation is used for the plotting position which involves sorting the block maximum values in ascending order and assigning an artificial probability as follows [14]:

$\mathrm{p}_{\mathrm{i}}=\frac{\mathrm{i}}{\mathrm{N}+1}$

A key condition with such an approach for extreme value estimation of bridge action effects is that the chosen structural element is undamaged and in the elastic region during the monitoring period. In the current approach no allowance is made for changes in traffic patterns or deterioration of the bridge in the 75 year return predictions.

\subsection{Preliminary fatigue safety verification}

Schläfli and Brühwiler [15] found that the reinforcement is the determinant fatigue element in the deck slabs of reinforced concrete bridges. In order to have a fatigue problem the rebar must experience stress ranges above the fatigue limit, therefore a preliminary measure of the fatigue safety can be obtained by direct measurement of the outer rebar on the tension face of a detail. Rainflow counting of the measured strains will provide histograms for damage calculation.

\section{Results and discussion}

\subsection{Monitoring duration}

The monitoring duration of action effects until stability of the maximum values was found to be highly dependent on the structural element's orientation. Fig. 6 illustrates how daily maximum strain measurements on elements in the lateral direction (S1a to S2c) are found to converge much slower to a limiting value than those in the longitudinal direction (SL1/SL2). The longitudinal measurements include both tension (positive) and compression (negative) response while the transverse bars only see significant tension effect. 30 days were required to achieve stability in the longitudinal rebar measurements while slight increases where still occurring in the transverse sensor (S1a/S1b) measurements even after 185 days. Transverse bars are influenced predominantly by the 
axle loads and lateral vehicle position, while the longitudinal behaviour is governed mainly by the gross vehicle weight (GVW) and hence a shorter time to stability. This effect can be further explained by examination of the simulated influence surfaces in Fig. 6(c/d). The surfaces are the response at the sensor location to a one tonne wheel load at any point on the bridge deck slab. The S1a transverse sensor location exhibits a very local influence surface peak in the longitudinal (X) direction while the SL1 influence surface for a longitudinal bar is much more gradual.

(a)

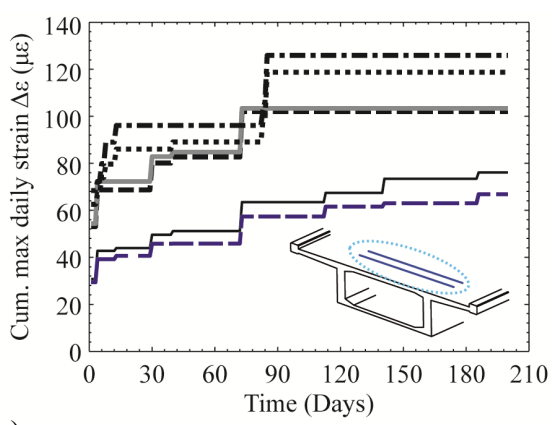

(c)

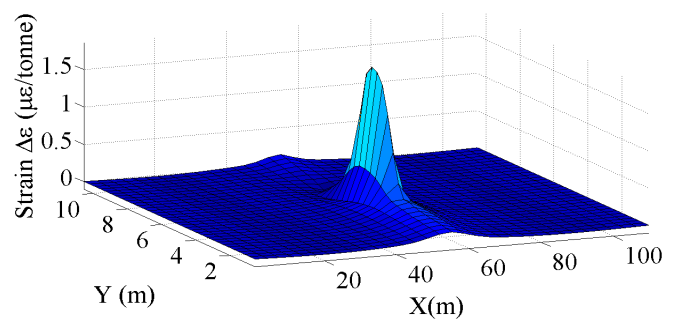

(b)

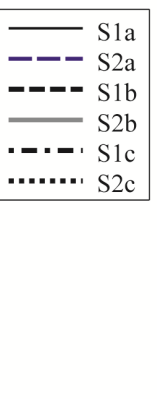

(d)
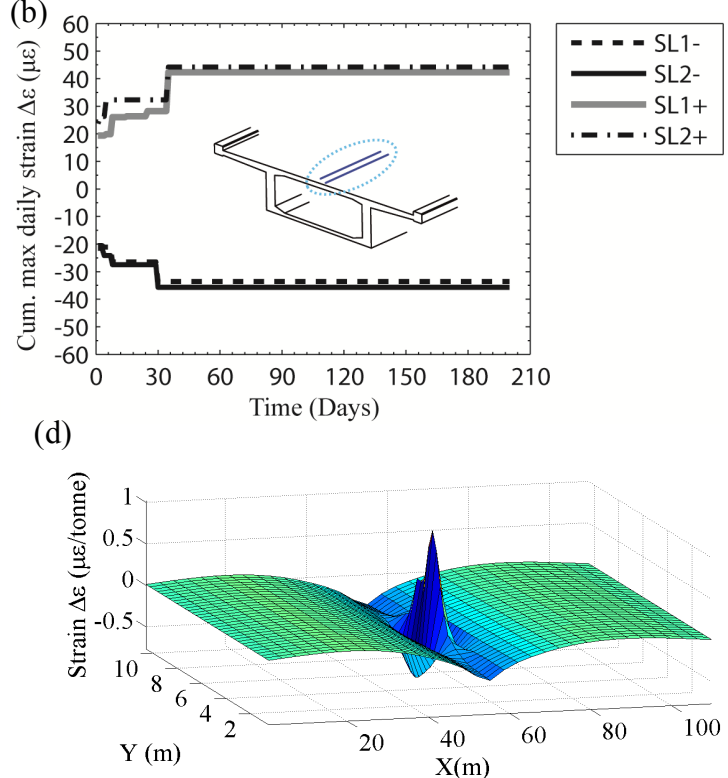

Fig. 6: Top - Cumulative max live load strains in transverse (a) and longitudinal rebars (b) Bottom - Simulated influence surface at sensors S1b (c) and SL1 (d)

\subsection{Extrapolation of measured data}

In this section the measurement series is used to extrapolate for the 75 year return period characteristic value of traffic action effect $E_{k}\left\{Q_{u p d a t e d}\right\}$. Maximum strain values for each sensor are selected based on 1 day block intervals. The thermal effect is removed as described in section 2.5 as the focus is on vehicle effects. Daily temperatures vary according to the season and would be likely to violate the assumption that the data has a common distribution [13]. Fitting a GEV to daily block maximum data shows good agreement between the measurements and the models as presented in Fig. 7. The pronounced curvature in Fig. 7(a) is indicative of the Weibull EVD form and can be verified as the value of $\xi<0$ (Table 1) as previously demonstrated for traffic load effects by other researchers including [16].

(a)

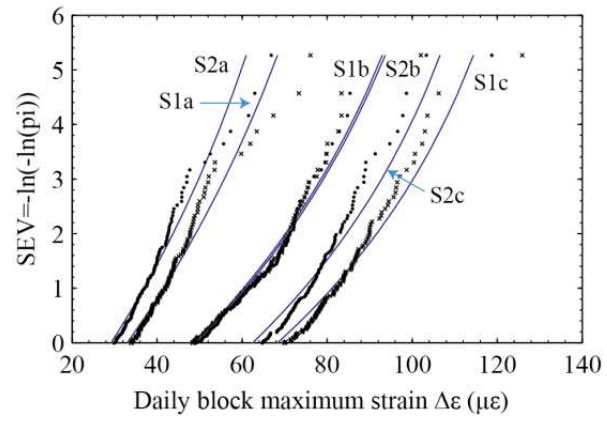

(b)

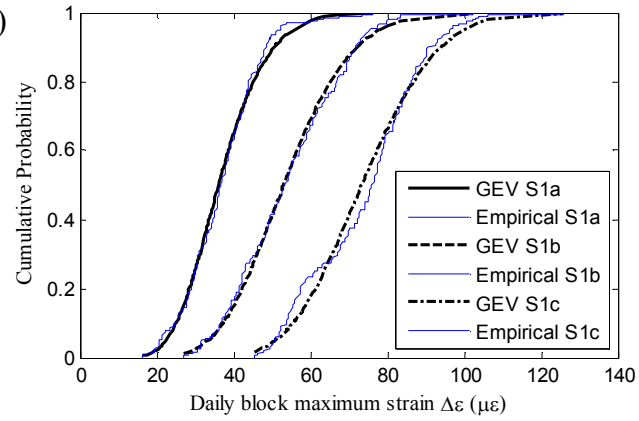

Fig. 7: (a) GEV fit versus measured data for both transverse rebars and; (b) GEV CDF for one transverse rebar

Fig. 8 illustrates the extreme value prediction based on various increasing monitoring periods up to 200 days. It can be seen that extrapolation to an extreme value based on a block maximum approach for the transverse rebars is highly sensitive to the measurement period. For example, a 90 day 
measurement period provides a larger 75 year return value. The monitoring regime will be continued beyond one year to investigate this further. In figure 8(b) the effect of the monitoring period on the GEV parameters is shown for sensor $\mathrm{S} 1 \mathrm{~b}$; with the shape parameter $\xi$ (kMLE) having the highest variability for low measuring periods.

(a)

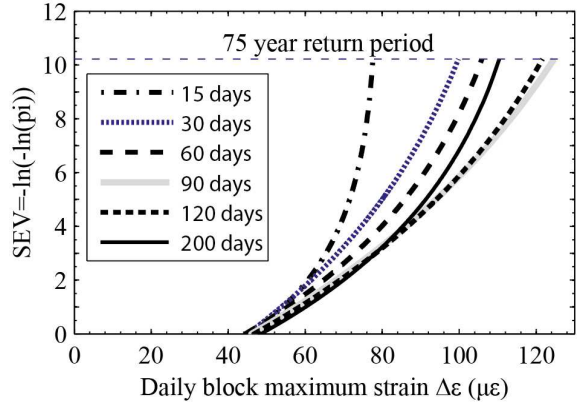

(b)

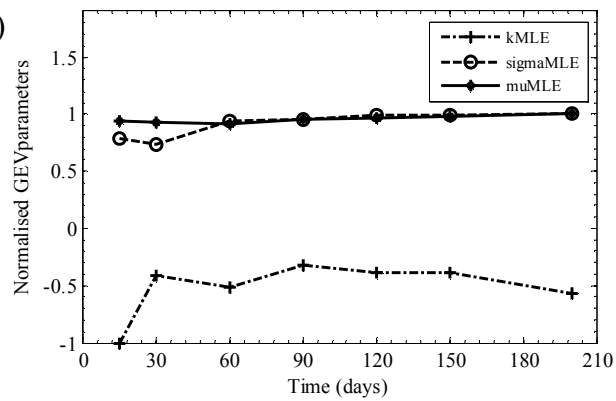

Fig. 8: (a) Extrapolation to a 75 year return period and; (b) Effect of monitoring period on GEV parameters for sensor $S 1 b$

In the simulation of extreme bridge load events, weekends can bias the results significantly due to low truck volumes. To avoid this, common practice is often to use an 'economic year' of 250 days [17]. However, for safety examination of existing bridges the weekends can be important. Although the volumes of heavy freight traffic at weekends are relatively low in this case study, rare severe load events can have a very large influence on the extreme predictions. For example day 73 in Fig. 9(a) occurred on a Sunday. This event alone significantly changed the overall behaviour of the GEV model and subsequent 75 year return period

\section{Table 1. Maximum likelihood GEV} parameters for Fig. 9(b)

\begin{tabular}{l|cccc}
\hline Case / Parameters & $\xi$ & $\sigma$ & $\mu$ & $\mathrm{z}(75 \mathrm{yr})$ \\
\hline With weekends & $-0,168$ & 12,7 & 48,7 & 110,4 \\
Without weekends & $-0,325$ & 11,3 & 55,5 & 89,2 \\
Without Sundays & $-0,281$ & 11,8 & 52,5 & 92,4 \\
\hline
\end{tabular}

(a)

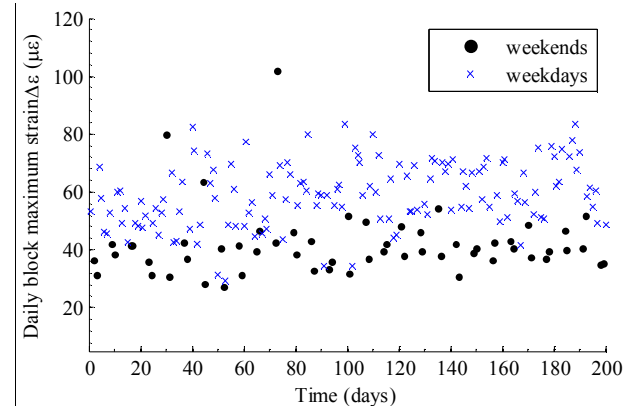
prediction (Fig. 9(b)). These rare weekend events are most likely due to special permission vehicles travelling on Sundays for safety reasons when traffic is quiet which will be further studied with associated WIM data. In this case the events are generally due to individual heavy vehicles rather than meeting events as the bridge features separate structures for each traffic direction.

(b)

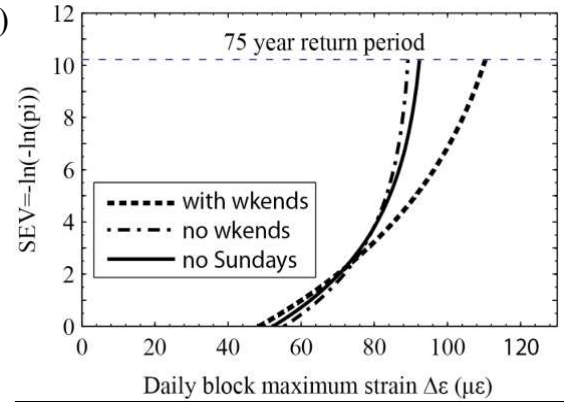

Fig. 9: (a) Comparison of daily maximums including weekends and; (b) effect of weekends on extrapolations for sensor $S 1 b$

For comparison Fig. 9(b) is based on a 75 year return period including 365 days per year for each case rather than modifying to remove weekends, etc. but this would only slightly affect the SEV. Future improvements will investigate extremes using a 'peaks over threshold' selection approach which recognises the fact that the second highest reading in one day could actually be higher than a maximum in another day.

\section{Conclusions}

The presented long-term high-frequency action effect measurements provide a unique insight into the behaviour of the monitored deck slab at an elemental level. Results from latest monitoring approaches have been combined with extreme value theory to provide a methodology for existing bridge element verification. The duration of monitoring allowed a better understanding of the 
timeframes needed to obtain stable measurements and the prediction of extreme values was very sensitive to the monitoring period. In addition, weekend monitoring and element orientation were found to be extremely important factors. While this particular structure shows no risk of failure, these techniques could be applied to local details identified as problematic by other means (e.g. calculation or visual inspection) for ULS verification. These techniques pave the way for more comprehensive safety verification procedure based on monitored data which is necessary for safely maintaining today's existing bridge stocks into the future.

\section{Acknowledgements}

The authors gratefully acknowledge the funding provided under the TEAM project, a Marie Curie Initial Training Network funded by the European Commission $7^{\text {th }}$ Framework Programme (PITNGA-2009-238648). They are also extremely grateful to the Swiss Federal Roads Authority (FEDRO) for allowing the opportunity to monitor the Morges Bridge presented in this study.

\section{References}

[1] European Comission, EU transport in figures. Statistical pocketbook 2011., Available at: http://ec.europa.eu/transport/publications/statistics/doc/2011/pocketbook2011.pdf, 2011.

[2] Siegert D., Estivin M., Billo J., Barin FX., and Toutlemonde F., "Extreme Effects of the Traffic Loads on a prestressed Concrete Bridge", International conference on heavy vehicles, Paris, 2008.

[3] Pircher M., Lechner B., Mariani O., and Kammersberger A., "Damage due to heavy traffic on three RC road bridges", Engineering structures, 2011, Vol. 33, No. 12.

[4] Treacy MA., and Brühwiler E., "A monitoring system for determination of real deck slab behaviour in prestressed box girder bridges", IABMAS 2012 - 6th International Conference on Bridge Maintenance, Safety and Management, Stresa, Italy, 2012.

[5] Wenzel H., Health monitoring of bridges, John Wiley \& Sons, West Sussex, 2009.

[6] National-Instruments, Smoothing Functions, Available at: http://zone.ni.com/reference/enXX/help/370859K-01/genmaths/genmaths/calc smoothfunctions/, 2012.

[7] Maljaars J., Steenbergen R., Abspoel L., and Kölstein H., "Safety Assessment of Existing Highway Bridges and Viaducts", Structural Engineering International, 2012, Vol. 22, No. 1, pp 112-120.

[8] Leander J., and Karoumi R., "Rate of convergence of measured stress range spectra", IABMAS 2012 - 6th International Conference on Bridge Maintenance, Safety and Management, Stresa, Italy, 2012.

[9] CEN, Eurocode 1: Actions on structures - Part 2: Traffic loads on bridges, European Committee for Standardization, 2003; p 164.

[10] Ghosn M., Sivakumar B., and Moses F., Modeling maximum live load effects on highway bridges, In Proceedings of the International Symposium on Life-Cycle Civil Engineering, IALCCE '08, Biondini, F., and Frangopol, D. Eds., CRC Press, Varenna, Italy 2008, pp 335341.

[11] Lipari A., O'Brien EJ., and Caprani CC., "A comparative study of a bridge traffic load effect using microsimulation and Eurocode load models", IABMAS 2012 - 6th International Conference on Bridge Maintenance, Safety and Management, Stresa, Italy, 2012.

[12] Brühwiler E., Vogel T., Lang T., and Lüchinger P., "Swiss standards for existing structures", Structural Engineering International, IABSE Zurich, 2012, Vol. 2012, No. 2, pp 275-280.

[13] Coles S., An introduction to statistical modeling of extreme values, Springer Verlag, 2001, p 208.

[14] Ang AHS., and Tang WH., Probability Concepts in Engineering Planning and Design: Volume I-Basic Principles, John Wiley \& Sons, New York, 1975, p 409.

[15] Schläfli M., and Brühwiler E., "Fatigue of existing reinforced concrete bridge deck slabs", Engineering structures, 1998, Vol. 20, No. 11, pp 991-998.

[16] Enright B., and O'Brien EJ., "Monte Carlo Simulation of extreme traffic loading on short and medium span bridges", Structure and Infrastructure Engineering: Maintenance, Management, Life-Cycle Design and Performance, 2012, Vol. 8, pp 1-16.

[17] Caprani CC., PhD Thesis, Probalistic analysis of highway bridge traffic loading, University College Dublin, 2005, p 396. 